\title{
Sign of decreased coronary blood flow from ST depression and PVCs during a left lower lobectomy of the lung in a patient with a left internal mammary arterial bypass graft
}

\author{
Jong-Kwon Jung, Sung-Il Hwang, Dae-Jin Lim, Seong-Jin Jeong, and Hyun Kyoung Lim \\ Department of Anesthesiology and Pain Medicine, Inha University Hospital, Incheon, Korea
}

Lung resection surgery in patients with a previous internal mammary artery (IMA) graft to coronary arteries is challenging, because the arterial graft may be injured during dissection of adhesions between the graft and the lung [1-3].

A 68-year-old male patient, diagnosed with a squamous cell carcinoma in the left lower lobe of the lung, required a left lower lobectomy. The patient (height, $160.4 \mathrm{~cm}$; weight, 73.5 $\mathrm{kg}$ ) was on the following medications: Aspirin, clopidogrel, losartan, amlodipine, and atorvastatin. Three years previously the patient had a coronary artery bypass graft surgery including a left IMA graft to the left anterior descending (LAD) artery. An electrocardiogram (ECG) showed normal sinus rhythm, occasional premature ventricular contractions (PVCs), and left ventricular hypertrophy. A preoperative transthoracic echocardiogram showed mild inferior wall hypokinesia. Preoperative coronary angiography revealed a patent left IMA to LAD artery flow.

After the patient arrived in the operating room, catheterization was performed on the patient's right radial artery. The patient was induced with $1-3.5$ vol\% sevoflurane after receiving 100 $\mathrm{mg}$ of propofol and $60 \mathrm{mg}$ of lidocaine by intravenous injection. Tracheal intubation using a double lumen endotracheal tube (37 Fr) was performed for one-lung ventilation 3 minutes after the patient received $50 \mathrm{mg}$ of rocuronium by intravenous injection. A central venous catheter was inserted into the right internal jugular vein due to the fear of graft pedicle injury and because we prefer the right internal jugular vein due to its straight course. At the start of the surgery, the patient's blood pressure was stable (mean systolic pressure, 100-120 mmHg; diastolic pressure, 40-60 $\mathrm{mmHg}$ ). After the thoracotomy, a severe adhesion between the upper lobe and the surrounding parietal pleura was noticed. The operator compressed the pericardium to control the bleeding using a sponge stick without considering the left internal mammary artery (LIMA) pedicle. During this period, ST-segment depression occurred, a series of PVCs appeared in about 5-6 seconds, and blood pressure decreased on the direct arterial pressure monitor (systolic pressure, 60 $\mathrm{mmHg}$; diastolic pressure, $30 \mathrm{mmHg}$ ). Phenylephrine (20 $\mu \mathrm{g} / \mathrm{cc}$ from a micro solution set) was dripped to increase blood pressure. The operator was notified immediately, the sponge stick compression was stopped, and the ECG and the blood pressure recovered to the previous state. Subsequent compression, as a provocation test, showed the same finding; therefore, it was concluded that the LIMA graft was tightly attached to the pericardium. To avoid further complications, Surgecel (Ethicon ${ }^{\mathrm{TM}}$, Johnson \& Johnson, Parsippany, NJ, USA) and Greenplast (Fibrin glue ${ }^{\mathrm{TM}}$, Green Cross, Seoul, South Korea) were used for bleeding control. The operation went successfully, and the postoperative course was uneventful.

A left upper lobectomy of the lung in patients with a previous LIMA to a LAD graft has been reported [1-3]. A patent LIMA pedicle injury can cause a myocardial infarction [3]. One case was reported in which a myocardial infarction due to systemic hypotension and proximal left subclavian artery stenosis occurred in a patient with a previous LIMA to a LAD graft [4]. This case shows that myocardial infarction can be induced by

Corresponding author: Hyun Kyoung Lim, M.D., Department of Anesthesiology and Pain Medicine, Inha University Hospital, 7-206, 3-ga, Shinheung-dong, Jung-gu, Incheon 400-103, Korea. Tel: 82-32-890-3968, Fax: 82-32-881-2476, E-mail: hkliman@inha.ac.kr

(c) This is an open-access article distributed under the terms of the Creative Commons Attribution Non-Commercial License (http:// creativecommons.org/licenses/by-nc/3.0/), which permits unrestricted non-commercial use, distribution, and reproduction in any medium, provided the original work is properly cited. 
decreased coronary blood flow due to direct injury of the LIMA pedicle or decreased flow of the artery that supplies the LIMA in a patent LIMA to a LAD graft in patients undergoing a left upper or lower lobectomy [3,4]. In this case, the LIMA pedicle could not be directly visualized due to the severe adhesion, but ST-segment depression and the series of PVCs resulting after compression strongly indicated that the blood vessels responsible for coronary flow were directly affected. Due to the risk of myocardial infarction by LIMA injury during the operation, we preoperatively prepared vasopressor (phenylephrine, norepinephrine, etc.) and a defibrillator and also considered the use of an intra-aortic balloon pump and possibly a regraft operation.

Conzen et al. [5] showed that patients receiving sevoflurane for off-pump coronary artery surgery had less myocardial injury than patients receiving propofol. Halogenated anesthetics have cardioprotective properties against ischemia and reperfusion injury by several mechanisms, including opening of the $\mathrm{K}_{\mathrm{ATP}}$ channels, adenosine receptor activation, and inhibition of the $\mathrm{Na}^{+} / \mathrm{K}^{+}$pump. As there was a high risk of LIMA injury in our surgery, sevoflurane was used as the main anesthetic agent, with the expectation that sevoflurane would provide cardioprotection against ischemia and reperfusion injury.

In conclusion, we could not confirm the location of the LIMA pedicle due to the severe adhesion. However, we believe that
ST-segment depression and the series of PVCs on the ECG were signs of decreased LIMA blood flow.

\section{Acknowledgements}

This work was supported by Inha University.

\section{References}

1. Halkos ME, Sherman AJ, Miller JI Jr. Preservation of the LIMA pedicle after cardiac surgery in left upper lobectomy. Ann Thorac Surg 2003; 76: 280-1.

2. Santini M, Fiorello A, Vicidomini G, Laperuta P. The use of LigaSure for preservation of a previous coronary artery bypass graft by using the left internal thoracic artery in a left upper lobectomy. J Thorac Cardiovasc Surg 2008; 136: 222-3.

3. Yazigi A, Jabbour K, Madi-Jebara S, Haddad F, Hayeck G, Tabet G. Myocardial infarction during left upper lobectomy in a patient with a LIMA graft. Anaesth Intensive Care 2009; 37: 331-2.

4. Dimas B, Lindsey JB, Banerjee S, Brilakis ES. ST-Segment elevation acute myocardial infarction due to severe hypotension and proximal left subclavian artery stenosis in a prior coronary artery bypass graft patient. Cardiovasc Revasc Med 2009; 10: 191-4.

5. Conzen PF, Fischer S, Detter C, Peter K. Sevoflurane provides greater protection of the myocardium than propofol in patients undergoing off-pump coronary artery bypass surgery. Anesthesiology 2003; 99 : 826-33. 